\title{
Efficiency of Respiratory Index in Determining Short-Term Prognosis of Multiple Trauma Patients: A Cross-Sectional Study
}

\author{
Golnar Sabetian, ${ }^{1}$ Shahram Paydar, ${ }^{2}$ Ali Rasti, ${ }^{3}$ and Zahra Ghahramani ${ }^{2,}{ }^{*}$ \\ ${ }^{1}$ Assistant Professor of Anesthesiology and Critical Care, Trauma Research Center, Shiraz University of Medical Sciences, Shiraz, Iran \\ ${ }^{2}$ Trauma Research Center, Shahid Rajaee (Emtiaz) Trauma Hospital, Shiraz University of Medical Sciences, Shiraz, Iran \\ ${ }^{3}$ Resident of Ophthalmology, Shiraz University of Medical Sciences, Shiraz, Iran \\ "Corresponding author: Zahra Ghahramani, Trauma Research Center, Shahid Rajaee (Emtiaz) Trauma Hospital, Shiraz University of Medical Sciences, Shiraz, Iran. Tel: \\ +98-7136360697, Fax: +98-7136254206, E-mail: ghahreman2@yahoo.co.nz
}

Received 2016 January 28; Revised 2016 October 15; Accepted 2017 January 06.

\begin{abstract}
Background: Being aware of trauma patients' conditions and predicting their outcome has always been of a great interest. To determine the state and prognosis of these patients, we should find ways to enable the timely identification of those with poor health and allow the physicians to treat them before the situation gets out of hand.

Objectives: The present study aimed at evaluating the efficiency of respiratory index (RI) in determining the short-term prognosis of multiple trauma patients in comparison with revised trauma score (RTS).

Methods: In this cross-sectional study, all multiple trauma patients who were admitted to emergency department (ED) of Shahid Rajaee hospital, Shiraz, Iran, during September and October 2013 were included. Demographic data and data regarding vital signs (blood pressure, heart rate, respiratory rate, GCS, and oxygen saturation), respiratory tract status, trauma type, blood gases, procedures performed in resuscitation room, and final outcome of the patients (discharge, disposition to general unit, intensive care unit, or operating room, and dying) were recorded using a predesigned checklist. Based on the collected data, RTS and RI were calculated for each patient and their correlation and the final outcome were evaluated.

Results: Evaluating 187 multiple trauma patients showed that 131 (70\%) patients had head injury, 78 (42\%) chest injury, 66 (35\%) abdominal injury, 49 (26\%) extremity injury, 27 (14\%) neck injury, and 4 (2\%) vascular injury. A significant correlation was seen between RI and RTS $(\mathrm{P}=0.024)$. RTS differentiated patients with good and poor health $(\mathrm{P}<0.05)$, while RI showed no significant correlation with patients' short-term final outcome.

Conclusions: Based on the findings of this study, RI cannot properly estimate short-term prognosis of multiple trauma patients, but it can be used as an independent factor in evaluating the severity of injury.
\end{abstract}

Keywords: Glasgow Coma Scale, Multiple Trauma, Patients, Prognosis, Respiratory Index

\section{Background}

Being aware of trauma patients' conditions and predicting their outcome has always been of a great interest. To determine the state and prognosis of patients, we should find ways to enable the timely identification of those with poor health and allow the physicians to treat them before the situation gets out of hand. Many indexes have been introduced as prognostic factors for predicting trauma outcome. Among these indexes are Glasgow coma score (GCS), revised trauma score (RTS), injury severity score (ISS), and its modified version new ISS (1). GCS was introduced in 1974 to help evaluate and record the severity of brain injury in trauma patients. This scale was considered as a base for determining the treatments used (2). ISS is a system for anatomical evaluation of patients with multiple trauma, which is linked to mortality, complications, duration of hospitalization, and other indicators of disease severity (3-5). Limitations of this system include errors in calculation and reaching equal scores in many injuries $(3,4)$. New ISS is an improved version of ISS that is able to predict the survival rate more accurately (6). RTS is used to predict the final outcome of the patients post-trauma and studies have demonstrated that this scale is useful in prediction of patient mortality (5, 7-9). Respiratory index (RI) $\left(\mathrm{RI}=\right.$ alveolar-arterial oxygen gradient $\left(\mathrm{P}(\mathrm{A}-\mathrm{a}) \mathrm{O}_{2}\right)$ normalized by $\mathrm{PaO}_{2}$ ), or the ratio of alveolar-arterial oxygenation gradient to arterial oxygenation is a scale that reflects the difference in oxygen concentration between lung alveoli and arterial blood, and its variations represent the differences in body oxygenation. RI rates rise by age, hepatic and cardiac diseases, sepsis, and respiratory diseases (10). This index can be used to evaluate respiratory function and severity of lung injuries (10-12). In trying to use this index, McFarlane et al. showed that this scale makes diagnosing embolism very unlikely and reduces the need for further evaluations (13). Also, in a similar study, Deutsch et al. expressed that this index cannot be an efficient screen- 
ing method for pulmonary embolism and that standard imaging methods should be used as soon as possible (14). It seems that this index can properly reflect the respiratory function and be used as a predicting factor for respiratory status in the vital hours post-trauma. Therefore, considering the number of multiple trauma patients and the importance of their timely treatment and resuscitation, in this study, we aimed at evaluating the efficiency of RI in determining the short-term prognosis of multiple trauma patients in an emergency department (ED) compared to RTS.

\section{Methods}

In the present cross-sectional study, all multiple trauma patients who were admitted to the emergency department of Shahid Rajaee hospital during September and October 2013 were evaluated. Patients hospitalized less than 6 hours, children under 14 years, and patients whose blood gases did not need to be measured were excluded. Demographic data and vital signs (blood pressure, heart rate, respiratory rate, GCS, and oxygen saturation), respiratory tract status, trauma type, blood gases, procedures performed in resuscitation room, and final outcome of the patients (discharge, disposition to general unit, intensive care unit, or operating room, and dying) were recorded using a predesigned checklist. RTS was calculated for each patient based on the scores obtained for GCS, systolic blood pressure, and respiratory rate per minute. RTS calculation method for multiple trauma patients is presented in Table 1. RI was calculated based on the standard formula using blood gas measurements. Average air and water vapor pressure in Shiraz, Iran, were considered 637 and $47 \mathrm{mmHg}$, respectively. Also, oxygen saturation and respiratory gas exchange ratio were $21 \%$ and 0.8 , respectively. After calculating RI and RTS rates, their correlation with one another and with the final outcome was evaluated to reach a method to determine the short-term prognosis of trauma patients. Short-term prognosis consisted of discharge, disposition to general unit, intensive care unit, or operating room, and dying. Data were analyzed by t test and ROC curve using SPSS Version 19. Significance level was considered to be $\mathrm{P}<0.05$. This study was approved by the ethics committee of Shiraz University of Medical Sciences. The researchers adhered to the principles of Helsinki declaration and protection of patient information.

\section{Results}

In this study, 187 multiple trauma patients, who were admitted to the ED during September and October 2013
Table 1. Revised Trauma Scale Calculations in Trauma Patients

\begin{tabular}{lccc}
\hline RTS Code & GCS & SBP & RR \\
\hline $\mathbf{4}$ & $13-15$ & $>89$ & $10-29$ \\
$\mathbf{3}$ & $9-12$ & $76-89$ & $>29$ \\
$\mathbf{2}$ & $6-8$ & $50-75$ & $6-9$ \\
$\mathbf{1}$ & $4-5$ & $1-49$ & $1-5$ \\
\hline $\mathbf{0}$ & 3 & 0 & 0 \\
\hline
\end{tabular}

Abbreviations: RR, Respiratory Rate; SBP, Systolic Blood Pressure.

were evaluated. Of the patients, 131 (70\%) had head injury, 78 (42\%) chest injury, 66 (35\%) abdominal injury, 49 (26\%) extremity injury, 27 (14\%) neck injury, and $4(2 \%)$ vascular injury. Table 2 demonstrates the patients' trauma information. Vital signs included blood pressure, heart rate, respiratory rate, GCS, oxygen saturation, and blood gas levels (Table 3). In this study, due to deficiency in the collected data, RTS was calculated for 143 out of the 187 patients and RI for 110. A significant correlation was obtained between RI and RTS ( $\mathrm{P}=0.024)$, RTS decreased as RI increased. Only 1 patient was discharged and 186 were hospitalized in different units or died. Of the patients, 66 were in good condition (1 was discharged and 65 were hospitalized in the general unit) and 121 had a poor condition (50 were hospitalized in intensive care unit, 57 were sent to operating room, and 14 died in the resuscitation room). RI and RTS relationship with patients' short-term prognosis was evaluated. Mean RTS was 11 for the discharged patients and 10.6 for those who were hospitalized, which is not significantly different. However, there was a significant difference between the patients with generally good and poor conditions $(\mathrm{P}<0.05)$.This means that RTS was able to predict the prognosis of patients with generally good and poor conditions. The best cutoff point was 10.5 , with $70 \%$ sensitivity and $74 \%$ specificity. Mean RTS was 10.25 for those who died and 10.7 for those who survived, which was not significantly different. Evaluation of the relationship between RI and the final outcome of the patients revealed no significant correlation with any of the studied outcomes. Figure 1 demonstrates the ROC curve for evaluating the ability of RI to differentiate the patients with generally good and poor conditions $(\mathrm{P}=0.420)$.

\section{Discussion}

Based on the findings of this study, it seems that RI compared to RTS, does not have the ability to predict shortterm prognosis of trauma patients and their outcome. The present study aimed to enable the better prediction of res- 
Table 2. Number and Percentage of Injuries Detected in Patients

\begin{tabular}{lc}
\hline Injury & No. $(\%)$ \\
\hline Vascular & $4(2.1)$ \\
\hline Extremities & $49(26.2)$ \\
\hline Abdomen & $66(35.3)$ \\
\hline Chest & $78(41.7)$ \\
\hline Neck & $27(144)$ \\
\hline Head & $131(70.1)$ \\
\hline
\end{tabular}

Table 3. Vital Signs and Blood Gas Level Recordings of Patients

\begin{tabular}{|lc|}
\hline Vital Signs & Mean $\pm \mathbf{S D}$ \\
\hline Systolic Blood Pressure, $\mathbf{~ m m} / \mathbf{H g}$ & $125 \pm 26$ \\
\hline Diastolic Blood Pressure, $\mathbf{~ m m / H g}$ & $78 \pm 17$ \\
\hline PR, Beat/min & $98 \pm 25$ \\
\hline RR, Resp/min & $22 \pm 10$ \\
\hline GCS & $10 \pm 4$ \\
\hline Oxygen saturation & $91 \pm 9$ \\
\hline Blood gas levels & \\
\hline \multicolumn{1}{|c|}{$\mathrm{HCO}_{3}$} & $22.5 \pm 18.4$ \\
\hline \multicolumn{1}{|c|}{$\mathrm{BE}^{\prime}$} & $-4.8 \pm 4$ \\
\hline $\mathrm{PCO}_{2}$ & $37.5 \pm 9.4$ \\
\hline $\mathrm{PO}_{2}$ & $54.5 \pm 33$ \\
\hline $\mathrm{PH}^{2}$ & $7.3 \pm 0.1$ \\
\hline
\end{tabular}

Abbreviations: PR. Pulse Rate; RR. Respiratory Rate.

piratory status of trauma patients admitted to the resuscitation room in Shahid Rajaee hospital and it showed that RI and RTS are significantly similar. As RI increased, RTS decreased, which indicated that RI can be an indicator of injury severity. Evaluating the relationship between RTS and final outcome of the patients revealed a significant correlation only with poor or good condition. In this case, RTS for 53 patients with good condition and 90 with poor condition showed that this scale can differentiate these 2 groups. Yet, it could not predict discharge or death of the patients. This can be due to the low number of cases in these groups. In evaluating the relationship between RI and final outcome, none of the studied outcomes showed any correlation, which indicates that although RI can be used as a factor indicating the severity of injury, it cannot accurately predict the short-term prognosis of multiple trauma patients. RI, or the ratio of alveolar-arterial oxygenation gradient to arterial oxygenation, is a scale that reflects the difference in oxygen concentration between lung

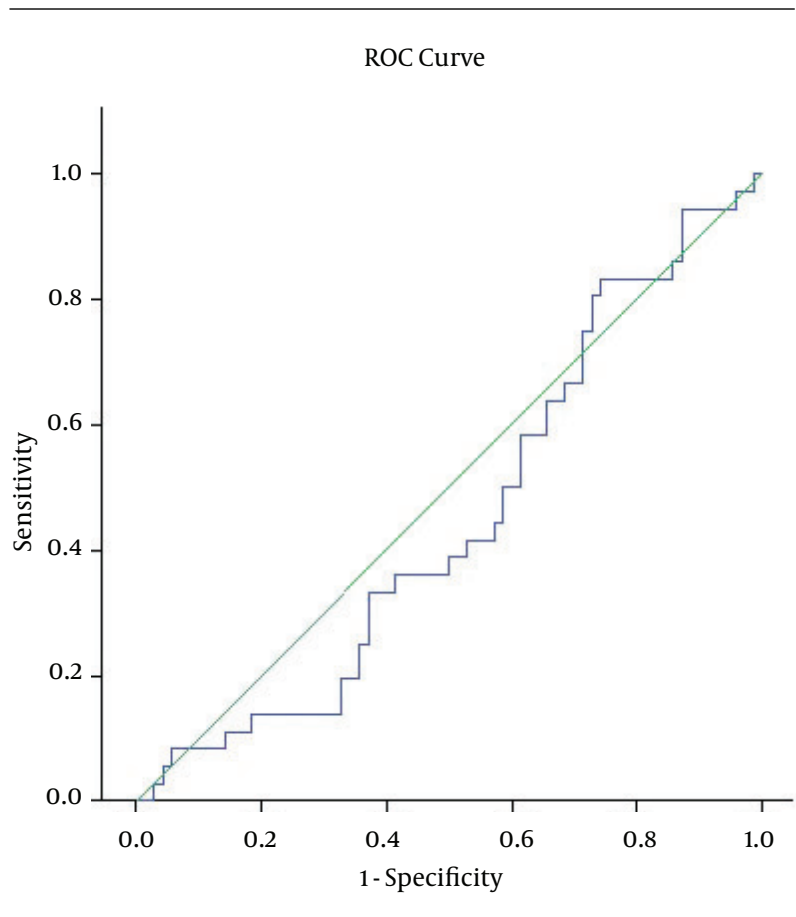

Figure 1. The Relationship Between Respiratory Index and Short-Term Prognosis of the Patients

alveoli and arterial blood, and its variations represent the differences in body oxygenation which can be calculated by knowing blood gas measurements (13). This index increases in sepsis, liver, cardiac, and especially respiratory illnesses and can, therefore, be used as a predictive factor for evaluating respiratory function and severity of respiratory injuries (10-12). In a study on 151 patients with respiratory and nonrespiratory sepsis, liver cirrhosis, and cardiac failure, RI was evaluated as an index for measuring respiratory oxygenation. As the results revealed, RI has the closest relationships with pulmonary shunt and venous blood oxygen content, which indicates that RI increases with the severity of the underlying illness. In patients with liver cirrhosis and extra-pulmonary sepsis, the reason for increased RI was ventilation perfusion mismatch, while in patients with pulmonary sepsis the reason was direct limitation in alveolar oxygen exchange, and in patients with cardiac failure it increased because of the a disproportionate decrease in mixed venous oxygen (10). In 1989, Laghi et al. studied 240 severe trauma patients, 88 of whom were affected by posttraumatic respiratory distress syndrome, and they found a significant correlation between RI/pulmonary shunt ratio and posttraumatic respiratory distress syndrome. This index showed a significant increase in patients with posttraumatic respiratory distress syndrome compared to trauma patients without 
respiratory distress syndrome, or those who were recovering from this syndrome (15). In another study in 1994, McFarlane et al. evaluated normal alveolar-arterial oxygen gradient in patients with suspected pulmonary embolism and compared it to compute tomography results as the gold standard. They expressed that normal alveolararterial oxygen gradient makes diagnosing embolism very unlikely and reduces the need for further evaluations in patients who had no history of pulmonary embolism or deep vein thrombosis (13). Also, in 2010, Deutsch et al. assessed the value of RI as a screening tool for embolism diagnosis in pregnant women and revealed that it is not an efficient and that standard imaging tools should be used for these patients (14). In a study, Chieregato et al. evaluated the relationship of difference in cerebral artery and vein $\mathrm{CO}_{2}$ pressure with the difference in arterial and venous oxygen in predicting cerebral ischemia in 55 severe cerebral injuries. They found that these evaluations alone are not specific enough to assess cerebral ischemia and better scales are needed (16). In 2012, Reed et al. designed a system aiming to predict death due to lower respiratory tract diseases in 4184 affected children. They expressed that their system effectively differentiates the children with lower respiratory tract diseases and can be used as a tool to determine mortality risk and severity of respiratory diseases in children (17). Then, Emukule et al. designed a modified version of this system for children under 5 years in 2014 and revealed that with an increase in this index, mortality rates also increased. They succeeded in predicting which children were more susceptible to death due to respiratory tract diseases (18). Therefore, we can conclude that although RI can be used as an injury severity factor due to its significant correlation with RTS, it cannot correctly estimate the short-term prognosis of multiple trauma patients. The results of this study dismiss using RI as a prognosis-predicting factor.

\subsection{Conclusion}

It seems that although RI can be used as an injury severity factor because of its significant correlation with RTS, it cannot correctly estimate the short-term prognosis of multiple trauma patients.

\section{Acknowledgments}

This article was extracted from Ali Rasti's thesis to achieve his general practitioner degree from Shiraz University of Medical Sciences. The researchers of this study wish to thank their colleagues in the emergency department of Shahid Rajaee hospital, Shiraz, Iran, and the research department of Shiraz University of Medical Sciences for their invaluable assistance in coducting this study.

\section{Footnotes}

Authors' Contribution: All authors met the 4 criteria by international committee of Medical Journal editors (ICMJE) to gain authorship. Design of the study, Shahram Paydar, Golnar Sabetian; drafting the article: Golnar Sabetian; final approval, Golnar Sabetian, Shahram Paydar, Ali Rasti, Zahra Ghahramani; administrative and technical support, Zahra Ghahramani; analysis and interpretation of data, Ali Rasti.

Conflict of Interest: Hereby, the authors declare that no conflict of interest exists regarding this research.

Financial Resources: No financial support has been received for this project.

\section{References}

1. Champion HR. Trauma scoring. Scand J Surg. 2002;91(1):12-22. doi: 10.1177/145749690209100104. [PubMed: 12075830].

2. Matis G, Birbilis T. The Glasgow Coma Scale-a brief review. Past, present, future. Acta Neurol Belg. 2008;108(3):75-89. [PubMed: 19115670].

3. Baker SP, O'Neill B, Haddon WJ, Long WB. The injury severity score: a method for describing patients with multiple injuries and evaluating emergency care. J Trauma. 1974;14(3):187-96. doi: 10.1097/00005373197403000-00001. [PubMed: 4814394].

4. Copes WS, Champion HR, Sacco WJ, Lawnick MM, Gann DS, Gennarelli $\mathrm{T}$, et al. Progress in characterizing anatomic injury. J Trauma. 1990;30(10):1200-7. doi: 10.1097/00005373-199010000-00003. [PubMed: 2213928].

5. Eichelberger MR, Gotschall CS, Sacco WJ, Bowman LM, Mangubat $\mathrm{EA}$, Lowenstein $\mathrm{AD}$. A comparison of the trauma score, the revised trauma score, and the pediatric trauma score. Ann Emerg Med. 1989;18(10):1053-8. doi: 10.1016/S0196-0644(89)80930-8. [PubMed: 2802280].

6. Stevenson M, Segui-Gomez M, Lescohier I, Di Scala C, McDonald-Smith G. An overview of the injury severity score and the new injury severity score. Inj Prev. 2001;7(1):10-3. [PubMed: 11289527].

7. Moore L, Lavoie A, Abdous B, Le Sage N, Liberman M, Bergeron E, et al. Unification of the revised trauma score. J Trauma. 2006;61(3):718-22. doi: 10.1097/01.ta.0000197906.28846.87. [PubMed: 16967013] discussion 722 .

8. Gilpin DA, Nelson PG. Revised trauma score: a triage tool in the accident and emergency department. Injury. 1991;22(1):35-7. doi: 10.1016/0020-1383(91)90158-B. [PubMed: 2030028].

9. Gabbe BI, Cameron PA, Finch $\mathrm{CF}$. Is the revised trauma score still useful? ANZ J Surg. 2003;73(11):944-8. doi: 10.1046/j.14451433.2003.02833.x. [PubMed: 14616576].

10. Sganga G, Siegel JH, Coleman B, Giovannini I, Boldrini G, Pittiruti M. The physiologic meaning of the respiratory index in various types of critical illness. Circ Shock. 1985;17(3):179-93. [PubMed: 4075505].

11. Yusa T, Nohara A, Sunagawa M. Relationship between plasma neutrophil elastase and respiratory index of patients who had undergone cardiac surgery with cardiopulmonary bypass. J Anesth 1995;9(4):324-8. doi: 10.1007/BF02479945. [PubMed: 23839880].

12. Gaillard M, Herve C, Mandin L, Raynaud P. Mortality prognostic factors in chest injury. J Trauma. 1990;30(1):93-6. [PubMed: 2296073].

13. McFarlane MJ, Imperiale TF. Use of the alveolar-arterial oxygen gradient in the diagnosis of pulmonary embolism. Am JMed. 1994;96(1):5762. doi: 10.1016/0002-9343(94)90116-3. [PubMed: 8304364]. 
14. Deutsch AB, Twitty P, Downes K, Parsons MT. Assessment of the alveolar-arterial oxygen gradient as a screening test for pulmonary embolism in pregnancy. Am JObstet Gynecol. 2010;203(4):373 e1-4. doi: 10.1016/j.ajog.2010.04.049. [PubMed: 20554265].

15. Laghi F, Siegel JH, Rivkind AI, Chiarla C, DeGaetano A, Blevins S, et al. Respiratory index/pulmonary shunt relationship: quantification of severity and prognosis in the post-traumatic adult respiratory distress syndrome. Crit Care Med. 1989;17(11):1121-8. doi: 10.1097/00003246-198911000-00006. [PubMed: 2791591].

16. Chieregato A, Marchi M, Fainardi E, Targa L. Cerebral arterio-venous pCO2 difference, estimated respiratory quotient, and early posttraumatic outcome: comparison with arterio-venous lactate and oxygen differences. J Neurosurg Anesthesiol. 2007;19(4):222-8. doi: 10.1097/ANA.0b013e31806589f6. [PubMed:17893572].

17. Reed C, Madhi SA, Klugman KP, Kuwanda L, Ortiz JR, Finelli L, et al. Development of the Respiratory Index of Severity in Children (RISC) score among young children with respiratory infections in South Africa. PLoS One. 2012;7(1):ee27793. doi: 10.1371/journal.pone.0027793. [PubMed: 22238570].

18. Emukule GO, McMorrow M, Ulloa C, Khagayi S, Njuguna HN, Burton D, et al. Predicting mortality among hospitalized children with respiratory illness in Western Kenya, 2009-2012. PLoS One. 2014;9(3):ee92968. doi: 10.1371/journal.pone.0092968. [PubMed: 24667695]. 